\title{
A Novel "Stylet" Device for Facilitating the Advancement of a Colonoscope
}

One of the main causes of difficulty when advancing a colonoscope is recurrent loop formation in the sigmoid colon. Techniques that have been developed to prevent this include the use of abdominal pressure, changing the patient's position, and the employment of a sliding tube [1]. These methods, however, have some disadvantages, such as the possibility of vagal reflexes, patient discomfort, mucosal injury, and discharge of liquid stool. To circumvent these potential problems, we have devised a new instrument, the "stylet". Here we report the usefulness of the stylet in colonoscopy.

The stylet used in our study is made of stainless steel. It has a diameter of $2.2 \mathrm{~mm}$ and is $133.6 \mathrm{~cm}$ in length overall, with a $6-\mathrm{cm}$ flexible section at the tip to protect the forceps channel (Figure [1]). In order to assess the stylet in colonoscopy, we studied a total of 200 consecutive patients who were undergoing colonoscopy, randomly dividing them into two groups of 100 patients. There were no significant differences between the two groups with respect to either gender or age at the time of enrollment and none of the patients had a history of abdominal surgery. In group A, when the tip of the colonoscope reached the splenic flexure of the colon, the sigmoidal loop was straightened and the stylet was inserted through the forceps channel. The insertion of the stylet stiffened the scope and allowed it to advance toward the transverse colon by keeping the sigmoid colon straight. The stylet was then withdrawn and the colonoscopic examination was continued as the scope advanced toward the cecum. In group B, the colonoscopy was performed in the usual manner, without using the stylet. In both groups, if recurrent loop formation occurred during the examination, abdominal pressure was applied and/or the patient's posistion was changed. We recorded the frequency of these maneuvers in the two groups.

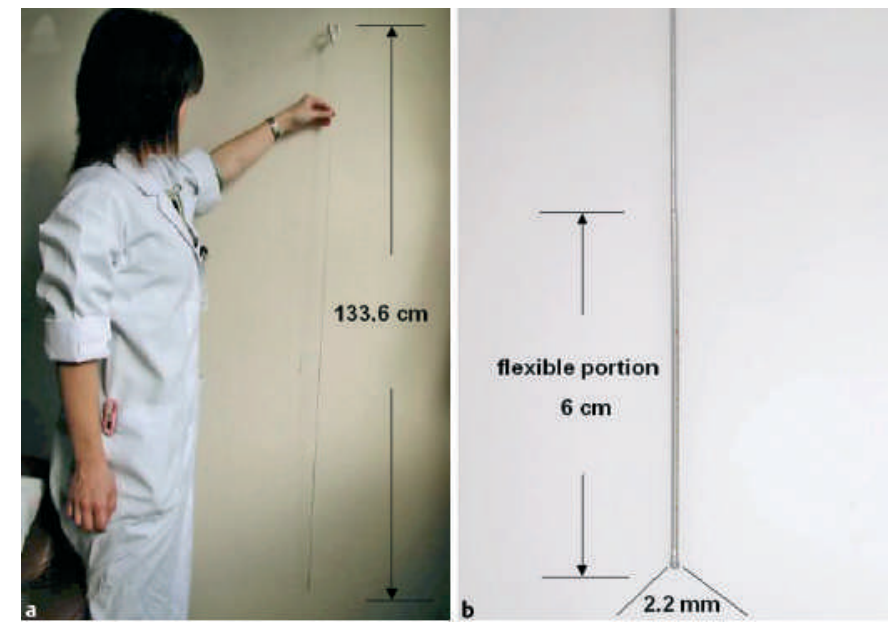

Figure 1 The "stylet" is made of stainless steel and is $133.6 \mathrm{~cm}$ in length (a). It is $2.2 \mathrm{~mm}$ in diameter, and at the tip there is a 6-cm flexible section, which protects the forceps channel (b).

There were only two patients in group $\mathrm{A}$ who required abdominal pressure and no patients in this group required changes in position. In contrast, in group B, 36 patients required abdominal pressure and 22 patients required changes in position; 18 patients required both abdominal pressure and changes in position. The differences between the two groups with regard to the requirement for abdominal pressure, the requirement for change in position, and the requirement for both maneuvers were all statistically significant $(P<0.05$, Mann-Whitney $U$ test). Insertion of the stylet did not cause any damage to the endoscopes. We experienced no difficulties in inserting the stylet and no complications occurred during the study.

This study showed that the frequency of application of abdominal pressure and of the need to change the patient's position during colonoscopy may be considerably reduced by the use of this stylet. Furthermore, total colonoscopy can be performed more safely, more quickly, and more cleanly than can be achieved with the use of a sliding tube.

\section{Competing interests: None}

M. Ogawa1, T. Nakatsu², T. Masaki', A. Morishita ${ }^{2}$, A. Muramatsu², H. Yoneyama ${ }^{2}$, N. Uchida', S. Kuriyama ${ }^{1}$ 1 Third Department of Internal Medicine, Kagawa University School of Medicine, Kagawa, Japan

2 Department of Gastroenterology and Internal Medicine, Saint Martin Hospital, Kagawa, Japan

\section{Reference}

${ }^{1}$ Makiishi H, Kitano A, Kobayashi T. A "sliding tube" method available for colonofiberscopy [in Japanese]. Gastroenterol Endosc 1972; 14: 95

\section{Corresponding Author}

\section{S. Kuriyama, M.D.}

Third Department of Internal Medicine Kagawa University School of Medicine 1750-1 Ikenobe

Miki-cho

Kita-gun

Kagawa 761-0793

Japan

Fax: $\quad$ +81-87-891-2156

E-mail: skuriyam@med.kagawa-u.ac.jp 